\title{
What is Social Prescribing?
}

\author{
Richard Kimberlee
}

\begin{abstract}
This article outlines research into social prescribing provision in one Clinical Commissioning Group area in England. Based on primary data collected from focus groups with social prescribing practitioners $(n=8)$, local council/Public Health employees $(n=6)$ and GPs $(n=4)$ and interviews with 40 social prescription practitioners and 22 patients from 23 interventions it shows that there is no clear and agreed definition of what constitutes social prescribing. Based on analysis of local practice this article delineates social prescribing interventions into four types: Signposting, Light, Medium and Holistic. It outlines the problems related to data collection around social prescribing interventions effectiveness but points to emerging evidence that suggests that SP holisticinterventions can contribute to improvement in patient wellbeing, reduction in anxiety and depression and attendance at GP surgeries.
\end{abstract}

Keywords: Holistic Social Prescribing, General Practice, Clinical Commissioning Group, Wellbeing

\section{BACKGROUND}

This article reports on research commissioned by a local Clinical Commissioning Group (CCG) in England. It was led by an Associate Director in Public Health. It was initiated to run in parallel with the decision to re-commission mental health services across the CCG area. Originally social prescribing (SP) was seen as part of the procurement process for mental health services for the city. However, this research was commissioned to provide advice on how best to commission SP in the future. To this end this research was conducted to offer the CCG guidance by providing: an outline of SP provision in the local area and an assessment of their evidence for effectiveness. Current interest in SP has arisen, not just in this CCG, but across the UK, because of two distinct issues: the increasing burden of mental health and other long-term conditions and the cost implications this poses for service provision and the growing crisis in general practice.

One in four people in the UK are known to suffer a mental health problem in the course of a year. It is acknowledged that within primary care around $30 \%$ of all consultations and $50 \%$ of consecutive attendances concern some form of psychiatric problem, predominantly depression or anxiety.[1][2] The cost of mental health problems to the economy in England have been estimated at $£ 105$ billion, and treatment costs are expected to double in the next 20 years. This is a personal cost that we all share. It is estimated that the economic costs of mental health are $€ 2000$ per annum for each European household.[3] Mental health remains high on the government's agenda. The No Health without Mental Health document, published by the Department of Health (2011), urged the development of a cross government approach to address the issue with a focus on outcomes for people with a mental illness as a way of developing and promoting solutions to reduce the burden. Although the picture varies across Europe, the emphasis in mental health services has moved towards the development of a more person-centred approach, based on principles of SP.[4]

This growth in mental illness is accompanied by mounting evidence that suggests that primary care services are under increasing strain. GP surgeries are seeing increasing numbers of patients presenting. GPs are not necessarily equipped to handle all the social and psychological 
burdens that patients present. The traditional model of service delivery is changing. GP has come a long way from a model where patients were examined in their living room. Today GPs usually practice in stand - alone surgeries and healthy living centres which offer an ever broadening range of services. Which services they develop and offer can vary across GP practices. But these changes and pressures coupled with complex reforms led Clare Gerada former Chair of the College of General Practitioners to conclude that general practice is in crisis![5]. Survey work commissioned by the College and undertaken by the Kings Fund revealed that: $85 \%$ of GPs believe their service was in crisis, nearly $50 \%$ of GPs believe they can no longer guarantee safe patient care, and that most GPs were conducting 40-60 patient consultations each day and working 11 hour days in the consulting room. Most GPs predicted that patients will have to wait longer for an appointment.[5]

With an aging population this burden is going to increase. It is anticipated that consultation rates will increase by $5 \%$ over the next 20 years. GPs also perceive that their patients were demanding better services and expect more. In particular younger patients are seen as less likely to grin and bear their ailments compared to older generations.[6]And unlike other health services primary care has no waiting list or referral criteria-they are forced to deal with the here and now in all its ramifications on a daily basis.[7]

With pressures on GPs growing some GPs are advocating and developing new approaches to their service delivery. This fresh approach includes SP. Dr Sam Everington, Chairman of Tower Hamlets CCG, has argued that GPs need assistance to manage their workload [8] and believes that GPs should be offered more incentives to develop partnerships to make health services work more effectively. The Chair of the CGP recently argued that GPs need all providers of health and social care, within a geographically aligned area to come together and pool resources.[9]And according to a retired GP from Bethnal Green Health Centre it requires commissioners and GPs to undertake a:a radical rethink on service provision, with perhaps less emphasis on classification and more on collaborative working practices. [10]

Part of this push to encourage primary care services to develop collaborative working is the realization that the burden of managing long-term conditions calls for a holistic approach. There are 15 million people in the UK living with at least one long-term condition. Typically this can include people who are repeat attendees in surgeries for which SP is increasingly seen as a potential solution. Recent Kings Fund Caring Research has led to a call for GPs to be more proactive and preventive in their approach. Thus, improving care for people with long-term conditions must involve a shift away from a reactive, disease-focused, fragmented model of care towards one that is more proactive, holistic and preventive, in which people with longterm conditions are encouraged to play a central role in managing their own care.[11]

Links between primary health care services and third sector organizations are often underdeveloped and require considerable time and patience to develop and evolve.[12] However some GPs have been turning to SP as a means of alleviating this burden. But what is SP? And how effective is it in addressing patient need and lessening burden? Unfortunately, there has been little research into the efficacy of SP. There is only one reported randomised control trial to assess SP cost effectiveness. However this study was undertaken a while ago and it only assessed an intervention that could be describe as SP medium (see below).In this study SP beneficiaries were seen to be less depressed and less anxious, but their care was more costly compared with routine care and their contact with primary care was not reduced.[13] 
The research outlined here therefore seeks to answer two questions.

○ What is SP?

○ Is it effective?

It was commissioned by an English CCG and sought to scope the range of SP practice in the CCG area.

\section{METHOD}

To understand what is meant by social prescribing I visited and interviewed service users, practitioners and commissioners involved with local well-being interventions (e.g. exercise gyms, IAPT and walking projects etc.). Those included in this research were self-defined SP projects. Exact identities of projects are not reported here, anonymity for SP participants was assured in order to facilitate open reflection and comment on the scale and impact of SP in the CCG area. This was important. Many providers of wellbeing interventions often found themselves in competition with each other to win funding to deliver and sustain their services. It was important to ensure that they felt the research was not an evaluation or assessment of their specific service but more an examination of the types of SP provision across the CCG area. Organizations delivering SP (particularly those in the third sector), run tight budgets and are acutely aware that there will be diminishing resources in the future and have subsequently developed a heightened sense of a need to showcase their work. In guaranteeing anonymity it meant participants in this research were able to provide open reflection on local provision.

Fieldwork took place in one CCG area between September and October 2013. The research took more time and resources than initially anticipated.This is partly because getting a GP perspective on SP proved challenging in terms of fitting around GPs' tight timescales which meant interviewing outside lengthy working days. Additionally, new SP providers not identified or knownby local Public Health officials began to contact me to ensure that their views were included in this process. Given the development and changes in local mental health services, SP providers demanded I broaden my research to include them. To this end I conducted focus groups with SP practitioners $(n=8)$, local council/Public Health employees $(n=6)$ and GPs $(n=4) .23$ self-defined SP providers were visited and interviewed. This included another 40 SP practitioners and 22 SP patients. The latter were not intentionally sought by the research but beneficiaries were frequently available when SP interventions were visited and they frequently engaged the researcher to discuss their experiences.

At a general level SP has emerged as a mechanism for linking people using primary care with support in their local community.[14][15] SP projects usually have a referral system in place and the SP element is often, but not exclusively delivered by a third sector partner. SP involves the creation of referral pathways that allow primary health care patients with non-clinical needs to be directed to local third sector organizations. Such schemes typically use community development workers or health workers with local knowledge or with skills to navigate locally. And they are formally linked to primary health care settings. SP also assist individual patients who present with social or psychological needs to access health resources and social support outside of the National Health Service. But they may also assist with patients who may present with a somatoform disorder i.e. where a patient has a mental disorder characterized by symptoms that suggest physical illness or injury - symptoms that cannot be explained fully by the individual. Or, where the GP believes a non-medical approach could achieve better outcomes. SP patients usually have not had a formal mental health diagnosis before. In fact they may not be suffering from a mental health disorder. But they are patients who present for which there are no obvious medical solutions. 
SP interventions are also seen as strengthening the links between health care providers and community, voluntary and local authority services. In these services there are potential solutions to the wider determinants of mental health, for example, leisure, welfare, education, culture, employment and the environment.[16] But these links between primary health care services and the third sector organizations are often underdeveloped and require considerable time to evolve.[17] In many SP projects the focus can often be on vulnerable and at risk groups and people with enduring and long term mental health problems. But what characterises SP more than anything else is that they are services that are purportedly offering a holistic approach [18] to a patient. And in many ways SP is a route to reducing social exclusion, both for disadvantaged, isolated and vulnerable populations in general, and for people with enduring mental health problems.[19]

Social prescribing creates a formal means of enabling primary care services to refer patients with social, emotional or practical needs to a variety of holistic, local non-clinical services.[20]

SP therefore seemingly aims to provide a referred patient with a holistic package of support tailored to their individual need.

SP packages can often be delivered through or alongside other opportunities e.g.: arts and creativity, physical activity, learning new skills, volunteering, mutual aid, befriending self-help etc. This could involve the SP worker offering an array of support around issues as diverse as: quitting smoking, addiction, relationship problems through to practical things like advice around housing, debt, legal advice, benefits or parenting problems. The Health Worker may also have additional skills around complementary therapies e.g. Reiki in the case of one SP project in the CCG area.

\section{RESULTS}

It is very clear from the literature and the interviews/focus groups undertaken for this research that there is no single, agreed understanding of what constitutes SP or what interventions/approaches can be called SP. Despite this the local interventions consulted in this CCG area continued to definethemselves as SP. In a lot of the literature including local policy documentation around the modernising mental health agenda the term SP was often used interchangeably with social intervention. In fact around the CCG area the term SP is applied to a variety of different interventions aimed at promoting wellbeing and/or health.

\section{Different models of social prescribing}

It is clear from my discussions with providers, practitioners, GPs and local authority employees that there was no agreement as to what they meant by SP. Focus group discussions tended to reach a reasoned understanding of what constitutes SP after considerable deliberation. But even then people did not necessarily agree on all aspects of SP or whether their experience of SP matched any broadly agreed criteria. To capture the range of SP interventions across the CCG area this article therefore outlines different models to explain and delineate between alternative self-defined SP interventions.

There were 57 GP surgeries accessed by patients from the CCG area, of which 12\% (n=7) had some form of SP intervention. These projects had evolved locally usually encouraged by an enthusiastic GP who felt that such approaches could offer more. All interventions definedthemselves as SP, however, most do not necessarily contain all the elements of holistic SP as outlined in the model below. They contain some elements and they may even be in a transition to another model. 


\section{SP as Signposting}

In this model the SP intervention is doing little more than signposting patients onto appropriate networks and groups who may assist an individual patient to address their wellbeing needs. All SP models have an element of signposting in their package. GPs can directly refer to the SP intervention and leave the patient to their own devices to access and follow through on the local wellbeing offerings available. Or the SP project may seek to address patient needs independent of the GP and will simply share the space of the practice but not necessarily have any regular or formal link with GPs. The activities that they may be referred too could include: a gym, a cooking project, peer support or a variety of counselling opportunities etc. The practice may not have a strong direct relationship with the SP project and there will be little or no follow-up and/or feedback. These projects will have only minimal evaluation of their outcomes. In the local CCG area this included a project called The Mirror. This was actually a tablet (IT) application that had been developed to help patients measure, visualise, and see the potential for change by allowing them to access online and offline networks of wellbeing support. Funding had come from different sources including a charity. It was being piloted in two GP practices with little evidence of its effectiveness. In essence it was a brokerage approach with the SP intervention highlighting gateways to other services.

In the CCG area there was arival on-line, free at the point of access, guide to thousands of health, wellbeing and community initiatives across the CCG and neighbouring CCG areas. It was run by a local umbrella group for third sector organizations. The website provided a mixture of links to frontline services and support to individuals, groups and organisations. It also had a unique Mental Health Employment Portal for patients to access.All these SP interventions had irregular or no links with GPs and appeared to simply co-exist with surgeries.

To be effective SP interventions depends on having good knowledge of what services are available in their local community. Mapping local, community groups and services into electronic health directories to facilitate signposting and referral helps SP projects to develop their knowledge base of what is available.[21] However to be effective SP signposting interventions often believe they need to employ local, trained, community health trainers to assist in the development and implementation of a signposting app' or on-line intervention. A recent evaluation of a SP signposting project revealed that: $70 \%$ of all referrals did engage with a link worker of which $91 \%$ set goals. Of those that were set goals $41 \%$ achieved their goals, but 59\% did not. Monitoring data shows $69 \%$ of patients, based on completed records, experienced an increase in SWEMWB score and that $64 \%$ have achieved an increase in confidence in managing their long-term condition.[22]

\section{Social Prescribing Light}

This is perhaps the most common form of SP. These are community and/or primary-care based interventions which refer at risk or vulnerable patients to a specific programme to address a specific need or to encourage a patient to reach a specific objective e.g. exercise on prescription, prescription for learning and arts on prescription.[23][24][25]In the CCG area there was a 'Wellbeing Prescription' project, which included walking activities organised under the auspices of a national volunteer charity. The initiative was new and had recently developed in response to a local need to address social isolation through walking.

Others included LinkAge which works with people aged 55+ in their local communities across the CCG area. Initially, the programme aimed to promote and enhance the lives of older people through a range of activities. This included fostering social awareness and encouraging older people to share their skills with both young people within the community and their families. In a sense it aimed to inspire older people and people within the local community to share time 
and experiences with older people who for one reason or another have become isolated. However it does not have any direct links with GP services although one of its hubs has been shown to significantly reduce isolation, promote well-being and increase physical activity rates.[26]

\section{Social Prescribing Medium}

The best example of this approach could be found outside of the CCG area. It was developed by senior partner who had been a GP in his town for 26 years. Like other SP initiatives their intervention included the employment of a Health Facilitator based in the practice. This role developed out of an exercise on prescription scheme developed by the local surgeries and the local Council ten years before. The health facilitator sees referred patients. Using Life Check and other tools the facilitator provided advice on exercise, nutrition, diet etc. They promote self-care using an on line Thought Field Therapy programme (rather like CBT) and also signpost to voluntary organisations or self-help groups for specific disease areas - e.g. patients with heart disease, diabetes and fibromyalgia or specific non-medicinal needs - e.g. a Knit and Natter group for people who are socially isolated, an amblers group for the overweight and unfit, creative writing, printing and book reading groups for patients needing directed activity/socialisation.

Although the project has a clear local remit in that it works within a distinct geographically defined neighbourhood and it is the product of joint partnership work,it does not obviously seek to address the beneficiary' needs in a holistic way instead it aims to address specific needs or behaviours identified by the GP.

\section{Social Prescribing Holistic}

Most of the SP interventions do not conform to this model of SP. This model of SP, I call: Social Prescribing Holistic. Interventions conforming to this model have usually evolved from the other models usually over a period of several years. They often co-locate with GP practices and have clear features:

- There is a direct primary care referral, usually from a GP practice, to an external SP provider. This is often formalised in terms of a letter, form, an on-line application or even a telephone call.

- The SP provider has a clear local remit and draws on local knowledge of local services and networks to connect patients to important sources of support and aid.

- The SP intervention has usually been developed and sustained jointly over time and in its present form represents a product of joint partnership work between the primary care provider and the SP provider.

- The SP provider addresses the beneficiary's needs in a holistic way. A patient may be referred to a SP project to improve e.g. diet, but in doing so the SP project will look at all patient needs and may offer support in terms of e.g. budgeting, nutrition, addiction, loneliness, access to employment etc.

- There are no limits to the number of times a patient is seen on a SP intervention. Time parameters may be set but the number of sessions offered can be more or less depending on the patient's needs discovered in the holistic approach.

- SP interventions seek to improve beneficiary' wellbeing. They may not necessarily initially be concerned with addressing mental health issues (although some are). A lot of patients who attend SP interventions have undiagnosed mental health issues. Although in adopting a holistic approach the SP project may delineate the mental health needs of the beneficiary and these will be addressed or sometimes the patient may be referred on to mental health services. 
SP holistic projects are adopting a holistic and preventive approach and aim to work with patients with long-term conditions. They encourage patients to play a central role in managing their own care. And it was very clear that they have emerged from organic partnerships that have independently developed between (usually a few) GPs in practice and their local third sector partner to address the perceived wellbeing needs that they both identify. They have evolved over time and sometimes from SP projects that could be previously described assignposting,light and medium.

\section{Effectiveness}

All SP projects present a lot of qualitative evidence to demonstrate the transformative effect their SP interventions have on beneficiary' lives. Their impact should not be underestimated. In adopting a holistic approach the complexity of the challenges addressed and the achievements they attain can be effectively demonstrated. GPs interviewed here believe that their SP holistic projects are making a real impact on the patients they refer.

We have seen how hard it is to engage some people, and how much patience and time some people need. In health we are often quick to judge, slow to listen, and feel too busy to care in the way we would want to. Having ****** (a local SP intervention) as a partner in our striving to deliver good care for our registered population is like having an extra pair of arms. The team are amazing in their resourcefulness and we are very much richer for the work they are doing.(A local GP)

Quantitative evidence deploying robust methodologies to demonstrate effectiveness were harder to find. Data monitoring of SP interventions were veryunder developed. Reasons expressed included: limited resources, cultural pressures, resistances from SP staff and patients unwilling to complete questionnaires etc.However two of the SP holisticinterventionsdiscovered in this research had understood the importance of the need for consistent data collection for monitoring and evaluation purposes and had invested in methodologies to measure impact.Data from one SP holistic project revealed that three months after a patient's $(n=70)$ induction on the SP intervention patients show statistically significant improvement in: PHQ9 ( $p=0.001)$, GAD7 $(p=0.001)$, Friendship Scale $(p=0.001)$, ONS Wellbeing (item range $\mathrm{p}=0.05$ through to $\mathrm{p}=0.001$ ) measures and IPAQ items for moderate exercise.Analysis of GP contact times $(n=37)$ also suggested that for 6 in 10 SP holisticpatients there is a reduction in their GP attendance rates in the 12 months post SP referral compared to the 12 months period prior to the referral. For $26 \%$ of beneficiaries it stayed the same and for $14 \%$ it actually increased.

It is hard to make cost comparisons across SP projects. Particularly inter-SP models. Even intra-model comparisons are fraught with difficulty. The organic development of allholistic SP projects makeseach SP intervention unique. Each has a different focus and they have evolved in time to meet varying local need. Amongst the holistic SP projects there are differences in the number and type of staff recruited. One model relies on a full time Health Worker with supporting volunteers. Another works with a male and a female Health Worker to deliver gender assigned one-to-one support. These cost variances also apply to fixed costs; some projects receive benefit in kind support in terms of having free accommodation and telephone access. One GP practice supports their SP intervention by covering these costs which are a great benefit to the provider. Other projects rely on external funding to cover their costs and sustain their work often from charitable (non-NHS) sources. But, by simply looking at the staffing costs/patient supported there was a range of cost effective ratios from: $£ 223.74$ to $£ 833$ for each patient supported by a SP holistic projects. 
But, it is very clear SP practitioners believe success is not simply about achieving positive outcomes like: improved well-being, a return to work or training etc.instead they see their intervention is about addressing embedded and unaddressed/undiagnosed issues like: agoraphobia brought on by abusive neighbours, addiction, obesity etc. In this sense SP interventions were seen as preventative in that practitioners believe they prevent patients from spiralling down to worse scenarios. In a recent review of the economic costs involved in mental health prevention the importance of intervening to prevent worse outcomes has been emphasised.[28] Simply looking at non-fatal suicide events it is estimated that costs are averted to $£ 66,797$ per year per person of working age where suicide is delayed. Figures vary depending on the means of the suicide attempt. $14 \%$ of costs are associated with A\&E attendance and medical or surgical care; but more than $70 \%$ of costs are incurred through follow-up with psychiatric inpatient and outpatient care.[28] Clearly suicide prevention is a potential saving that should be considered. One patient saw SP as saving their life.

I don't want to remember the past...it's not that I don't want to remember it....its like I got rid of it.... I was in my house.... I was on a tag everyday.... My Mum was an alcoholic, she was suicidal, my sister was in and out of psychiatric wards, she sliced herself on a daily basis she has also sat down and watched one of her kids.........because they were all saying that you are a criminal we are going to class yourself as a criminal when really I was a sick criminal and needed help for my condition but like they were diagnosing me but like none of them were giving me help.(One SP Holistic patient)

\section{CONCLUSION}

This research into SP interventions in one CCG area shows SP interventions are quite diverse. After conducting focus groups with SP practitioners, local council/Public Health employees, GPs and interviews with 23 self-defined SP providers this article has developed a typology of SP interventions: signposting, light, medium and holistic.These typologies will assist GPs, commissioners and practitioners to understand the type of intervention they are considering for their practice. However there is diversity within these models and no SP intervention is the same. Amongst the holistic SP projects there are considerable differences in the number and type of staff recruited, their focus activity, the form of referral from the GP etc.

Evidence of impact is quite limited particularly for non-holistic interventions. However, before and after data from one SP holistic project suggests that their intervention helped to reduce anxiety, depression, social isolation, GP attendance, and increase wellbeing and moderate exercise amongst referred patients. Currently there are very few opportunities of sharing best practice and experience of SP in the CCG area to ensure its development as an option for supporting patients in primary care. However commissioners should be aware of the additional economic value provided through SP projects which include: harnessing volunteers, patients returning to employment and training and the uptake of child care responsibilities and enhanced community capacity. Engaging with SP may help GPs facing 'crisis' find alternative approaches to reduce the burden of patients who draw increasingly on their services. With demand increasing, this supply side solution offers an alternative approach to promoting patient wellbeing.

\section{References}

Kessler D, Lloyd K, and Lewis G. (1999) Cross-sectional study of symptom attribution and recognition of depression and anxiety in primary care, BMJ 318:436-39

Scottish Executive (2005) Doing well by people with depression: Evidence topractice - a guide to help develop services for the management of depression, Edinburgh: Scottish Executive. 
European Social Network (2011) Mental Health and Wellbeing in Europe: A person-centred community approach, ESN: Brussels.

European Social Network (2011)op. cit. p8

Gerada C. (2013) Final keynote speech to the Royal College of General Practitioners Annual conference, 3rd October 2013, Harrogate, London. Accessed 8th October 2013.

Beavers N. (2013) Social prescribing and community facing services may be the future for GPs, Primary Care Today, Autumn 2013.

Hardy J. (2013) Putting less emphasis on classifying mental disorders and more on collaborative working, BMJ, 2013;347:f5873.p347

Beavers, N. (2013) op. cit.p5

Gerada C. (2013)op. cit.

Hardy J. (2013) op. cit. p347

Coulter A, Roberts S. and Dixon A. (2013) Delivering better services for people with long-term conditions: building the house of care. Oct 2013. The King's Fund: London. www.kingsfund.org.uk/publications/delivering-betterservicespeople-long-term-conditions Accessed 17th October 2013

South J, Higgins T, Woodall J. and White S. (2008). Can social prescribing provide the missing link? Primary Health Care Research \&amp; Development, 9, pp 310-318. doi:10.1017/S146342360800087X.

Grant C, Goodenough T, Harvey I. and Hine C. 2000: A randomized controlled trial and economic evaluation of a referrals facilitator between primary care and the voluntary sector. BMJ, 320, 419-23.

Brown M, Friedli L. and Watson S. 2004: Prescriptions for pleasure, Mental Health Today, June 20-23, 2004.

Scottish Development Centre for Mental Health (2007) Developing Social Prescribing and Community Referrals for Mental Health in Scotland, Edinburgh: The Stationery Office.

Scottish Development Centre for Mental Health (2003) Building Community Wellbeing An Exploration of themes and issues. Project report to the Scottish Executive, Edinburgh: The Stationery Office.

South J. et al (2008) op. cit.

Brandling J. and House W. (2007) Investigation into the feasibility of a social prescribing service in primary care: a pilot project. University of Bath and Bath and NE Somerset NHS.

Evans R, Henderson M, Lunney M. \& Thompson J. (2011) North Tyneside Social Prescribing Hub: Mental Wellbeing Impact Assessment.

Brandling J. and House W. (2007) p15 op. cit.

Coulter et al (2013) p16 op. cit.

ERS Research and Consultancy (2013) Newcastle Social Prescribing Project: Final Report August 2013. http://www.healthworksnewcastle.org.uk/wp-content/plugins/downloads-

manager/upload/Social\%20Prescribing\%20Evaluation\%20Report\%20August\%202013\%20Final.pdf, Accessed22nd October 2013.

Friedli L. and Watson S. (2004) Social prescribing for mental health. Durham: Northern Centre for Mental Health.

Tyldesley R. and Rigby T. (2003) The Arts on Prescription Postnatal Depression Support Service: an evaluation of a twelve week pilot Stockport.

Millin S. (2003) What are the Barriers for Arts on Prescription? City University: unpublished MA thesis.

Kimberlee R. and Means R. (2012) Assessing the Impact of the LinkAge hub in Whitehall and St.George, Bristol: Briefing Report, Bristol: UWE.

Brandling J. and House W. (2009) Social prescribing in general practice: adding meaning to medicine, British Journal of General Practice, 59(563): 454-456.

Knapp M, McDaid D, \& Parsonage M. (Eds.). (2011). Mental health promotion and mental illness prevention: The economic case. London: Department of Health. 